\title{
A Closed Form Frequency Estimator for a Noisy Sinusoid
}

\author{
H. C. So \\ Department of Computer Engineering \& Information Technology \\ City University of Hong Kong, Tat Chee Avenue, Kowloon, Hong Kong \\ e-mail: ithcso@cityu.edu.hk
}

\begin{abstract}
Based on the linear prediction property of sinusoidal signals, a closed form unbiased frequency estimator for a real sinusoid in white noise is proposed. The frequency estimate derives from minimizin $g$ a constrained least squares cost function and it is simple to implemen $t$ either in batch or recursive mode. The relationship of the proposed method with the well-known Pisarenko harmonic decomposer (PHD) is also examined. Computer sim ulations are included to contrast the estimator performance with the PHD as $w$ ell as the Cram ér-Rao lower bound.
\end{abstract}

\section{INTR ODUCTION}

Frequency estimation of sinusoidal signals in noise is a frequently addressed problem in the signal processing literature and it has a wide variety of applications such as angle of arrival estimation, demodulation of frequency-shift keying (FSK) signals, speech analysis and Doppler rate estimation [1]-[4].

For a noisy complex sinusoid, it is well known that [1] the maxim um likelihood (ML) estimate of frequency is obtained from the periodogram maximum. Kenefic and Nuttall [5] have extended the problem to ML frequency estimation of a real tone and the optim um estimator maximi zes a highly nonlinear and multimodal cost function. For both cases, ML methods in volve extensive computations and this will be prohibited in applications where rapid frequency estimation is required. Apart from the ML approac h, other frequency estimation techniques [3] include notch filtering, Capon methods, linear prediction, Yule-Walker methods and subspace-based approach. Among the subspace-based methods, the Pisarenko harmonic decomposer (PHD) [6] is of historical interest because it was the first to exploit the eigenstructure of the covariance matrix, and its performance has been extensively studied [6]-[10]. Interestingly, the PHD for a single real sinusoid can be implemented in a very simple way [11]-[12].

In this paper, we will focus on estimating the frequency of a real-valued tone in white noise. In Section II, the linear prediction approach for single sinusoidal frequency estimation using least squares (LS) is first reviewed. It is observed that the standard LS cost function for a real-valued tone should be minimi zed subject to constraints in order to attain unbiased frequency estimation. We then develop a modified LS estimator which leads to unbiased as well as closed form frequency estimation. The proposed estimator is computationally attractive and on-line computation is allowed. Its relationship with the Pisarenko's method is investigated in Section III. Numerical examples are presented in Section IV to evaluate the performance of the proposed algorithm by comparing with the PHD. It is also shown that the estimator variance can approach the CramérRao lower bound (CRLB) when the observation length is short and noise level is small. Finally, conclusions are drawn in Section V.

\section{ESTIMATOR DEVELOPMENT}

The noisy discrete-time measurements of the sinusoid are represented as

$$
\begin{aligned}
x(n)=\alpha \cos \left(\omega_{0} n+\phi\right) & +q(n), \\
n & =0,1, \ldots, N-1
\end{aligned}
$$

where the noise $q(n)$ is assumed to be a zero-mean white Gaussian process with variance $\sigma_{q}^{2}$ while $\alpha, \omega_{0} \in(0, \pi)$ and $\phi \in[0,2 \pi)$ are unknown constants which denote the tone amplitude, frequency and phase, respectively. The integer $N$ represents the number of available measurements. Without loss of generalit y, the sampling period is normalized to be unity. The task is to estimate $\omega_{0}$ from the $N$ samples of $x(n)$.

In the absence of noise, $x(n)$ is perfectly predictable from its past samples as:

$$
x(n)=2 \cos \left(\omega_{0}\right) x(n-1)-x(n-2)
$$

Based on this linear prediction property, we can easily develop a LS estimator for $\omega_{0}$ as follows,

$$
\hat{\omega}_{0}=\arg \min _{\hat{\omega}_{0}}\left\{\sum_{n=2}^{N-1} e^{2}(n)\right\}
$$

where $e(n)$ is an error function of the form

$$
e(n)=x(n)-2 \cos \left(\hat{\omega}_{0}\right) x(n-1)+x(n-2)
$$


and $\hat{\omega}_{0}$ denotes the estimate of $\omega_{0}$.

However, this estimator is biased and it can be easily verified from the mean square value of $e(n), E\left\{e^{2}(n)\right\}$, which is calculated as

$$
\begin{aligned}
E\left\{e^{2}(n)\right\}= & 4\left(\cos \left(\hat{\omega}_{0}\right)-\cos \left(\omega_{0}\right)\right)^{2} \sigma_{s}^{2} \\
& +2\left(2+\cos \left(2 \hat{\omega}_{0}\right)\right) \sigma_{q}^{2}
\end{aligned}
$$

where $\sigma_{s}^{2}=\alpha^{2} / 2$ denotes the tone power, because the noise component is a function of $\hat{\omega}_{0}$. To remove the effect of noise, a number of methods [12]-[14] has been proposed to minimize $\sum_{n=2}^{N-1} e^{2}(n)$ or $E\left\{e^{2}(n)\right\}$ subject to the noise component of (5) equals a constant and they can be referred to as the unit-norm constraint approach.

Based on the unit-norm technique, we employ a modified error function, $\zeta(n)$, which has the form:

$$
\zeta(n)=\frac{e(n)}{\sqrt{2\left(2+\cos \left(2 \hat{\omega}_{0}\right)\right)}}
$$

Using (5) and (6), it can be shown that the performance surface $E\left\{\zeta^{2}(n)\right\}$ has the minimum value of $\sigma_{q}^{2}$ at $\hat{\omega}_{0}=$ $\omega_{0}$. As a result, we form a new LS cost function for unbiased frequency estimation:

$$
J_{N}\left(\hat{\omega}_{0}\right)=\sum_{n=2}^{N-1} \frac{e^{2}(n)}{2\left(2+\cos \left(2 \hat{\omega}_{0}\right)\right)}
$$

It is worthy to note that (7) can be considered as an alternate form of the modified least squares cost function suggested in [15] but a key difference is made by constraining the parameter vector as a function of the estimated frequency. In so doing, unlike [15], the solution can be directly derived.

Differentiating $J_{N}\left(\hat{\omega}_{0}\right)$ with respect to $\hat{\omega}_{0}$ and then setting the resultant expression to zero, we get a very nice equation

$$
\begin{gathered}
\sum_{n=2}^{N-1} e(n)\left((x(n)+x(n-2)) \cos \left(\hat{\omega}_{0}\right)+x(n-1)\right)=0 \\
\Rightarrow 2 A_{N} \cos ^{2}\left(\hat{\omega}_{0}\right)-B_{N} \cos \left(\hat{\omega}_{0}\right)-A_{N}=0
\end{gathered}
$$

where

$$
A_{N}=\sum_{n=2}^{N-1}(x(n)+x(n-2)) x(n-1)
$$

and

$$
\begin{aligned}
B_{N}= & x^{2}(N-1)-x^{2}(N-2)-x^{2}(1)+x^{2}(0) \\
& +2 \sum_{n=2}^{N-1} x(n) x(n-2)
\end{aligned}
$$

The frequency estimate is then computed from one of the roots of (8):

$$
\hat{\omega}_{0}=\cos ^{-1}\left(\frac{B_{N}+\sqrt{B_{N}^{2}+8 A_{N}^{2}}}{4 A_{N}}\right)
$$

Substituting the expected values of $A_{N}$ and $B_{N}$, namely, $(N-2) \alpha^{2} \cos \left(\omega_{0}\right)$ and $(N-2) \alpha^{2} \cos \left(2 \omega_{0}\right)$, respectively, into (11), we get $\hat{\omega}_{0}=\omega_{0}$ and this implies that the proposed method gives an unbiased estimate of $\omega_{0}$.

Apart from the batch formula of (11), it is often desirable to compute the frequency estimate on a sample-b ysample basis particularly for real-time processing. From (9) and (10), it can easily be shown that $A_{N}$ and $B_{N}$ can be computed recursively using

$$
A_{N}=A_{N-1}+x(N-2)(x(N-3)+x(N-1))
$$

and

$$
\begin{aligned}
B_{N}= & B_{N-1}+x^{2}(N-3)-2 x^{2}(N-2) \\
& +x^{2}(N-1)+2 x(N-3) x(N-1)
\end{aligned}
$$

This on-line realization is simple to implem ent and is computationally attractive because only 8 additions, 7 multiplications, 1 division, 1 root operation and 1 arccos function are needed for each sampling interval.

\section{COMP ARISON WITH PHD}

It is well known that the estimate of $\omega_{0}$ in the PHD is found from the eigenvector corresponding to the smallest eigenvalue of the following $3 \times 3$ covariance matrix $[7]-[8]$ :

$$
\mathbf{R}=\left[\begin{array}{lll}
r_{0} & r_{1} & r_{2} \\
r_{1} & r_{0} & r_{1} \\
r_{2} & r_{1} & r_{0}
\end{array}\right]
$$

where $r_{k}$ is the sample covariance which has the form

$$
r_{k}=\frac{1}{N-k} \sum_{n=0}^{N-k-1} x(n) x(n+k), \quad k=0,1,2
$$

The eigenvector that corresponds to the smallest eigenvalue of $\mathbf{R}$ is given by

$$
\mathbf{v}=\left[\begin{array}{lll}
1 & \frac{-r_{2}-\sqrt{r_{2}^{2}+8 r_{1}^{2}}}{2 r_{1}} & 1
\end{array}\right]
$$

and the PHD frequency estimate, denoted by $\hat{\omega}_{0}^{o}$, is computed as [10]-[11]

$$
\hat{\omega}_{0}^{0}=\cos ^{-1}\left(\frac{r_{2}+\sqrt{r_{2}^{2}+8 r_{1}^{2}}}{4 r_{1}}\right)
$$


Expressing $A_{N}$ and $B_{N}$ in terms of $r_{1}$ and $r_{2}$ yields

$$
\begin{aligned}
A_{N}= & 2(N-2) \cdot \\
& \left(r_{1}+\frac{2 r_{1}-x(0) x(1)-x(N-2) x(N-1)}{2(N-2)}\right)
\end{aligned}
$$

$$
\begin{aligned}
\stackrel{\text { and }}{B_{N}}= & 2(N-2) \cdot\left(r_{2}+\right. \\
& \left.\frac{x^{2}(0)-x^{2}(1)-x^{2}(N-2)+x^{2}(N-1)}{2(N-2)}\right)
\end{aligned}
$$

Comparing (11) and (17)-(19), it is found that the second terms of (18) and (19) contribute to the difference between the proposed algorithm and the original PHD. An obvious advantage of (11) observed from (15) and (17) is that on-line implemen tation of the PHD will be much more complicated than the proposed technique. Since (11) is very similar to the Pisarenko's method, although a novel derivation is used, we call this estimator as reformed Pisarenko harmonic decomposer (RPHD).

\section{SIMULA TION RESUTS}

Computer sim ulations had been carried out to evaluate the frequency estimation performance for a single real sinusoid of the proposed algorithm. We compared its accuracy with those of the original PHD [10]-[11] as well as the CRLB. All simulation results provided were averages of 500 independent runs. The tone amplitude was set to $\sqrt{2}$ and $\phi$ was a constant uniformly distributed between $[0,2 \pi)$ at each trial, while different signal-tonoise ratios (SNRs), defined as $\sigma_{s}^{2} / \sigma_{q}^{2}$, were obtained by proper scaling the white Gaussian noise sequence.

Figure 1 shows the frequency variances of the RPHD and PHD as well the CRLB, for $\omega_{0} \in[0.05 \pi, 0.985 \pi]$ at $\mathrm{SNR}=20 \mathrm{~dB}$ and $N=10$. We observe that the RPHD outperformed the PHD in spite of the similarit $y$ in their algorithms. Moreo ver, the variances of $\hat{\omega}_{0}$ exceeded the CRLB by less than $4 \mathrm{~dB}$ for $\omega_{0} \in(0.2 \pi, 0.8 \pi)$ and it had the best performance at $\omega_{0} \approx 0.5 \pi$ where the degradation from the CRLB was approximately $1 \mathrm{~dB}$. This means that the proposed method is a sub-optim um frequency estimator for a wide range of frequency when the SNR is high and observation interval is short.

The above test was repeated for $N=400$ at $\mathrm{SNR}$ $=20 \mathrm{~dB}$ and the results are shown in Figures 2. It is seen that the RPHD was superior to the PHD even for a large sample size and the RPHD variances were less than those of the PHD by approximately $10 \mathrm{~dB}$ for $\omega_{0} \in(0.2 \pi, 0.8 \pi)$. Note that the curve of RPHD variance differed from that of Figure 1 and it had minimum values at $\omega_{0} \approx 0.28 \pi$ and $\omega_{0} \approx 0.72 \pi$. Figures
3 plots the frequency variances of the RPHD and PHD versus SNR at $N=10$ for $\omega_{0}=0.5 \pi$. We observe that the superiority of the RPHD over the PHD increased with SNR. In addition, the deviation between the RPHD variances and CRLB had minim um values of around $1 \mathrm{~dB}$ for $\mathrm{SNR} \geq 20 \mathrm{~dB}$. The frequency variances of the RPHD and PHD versus $N$ at $\mathrm{SNR}=20$ $\mathrm{dB}$ for $\omega_{0}=0.5 \pi$ are shown in Figure 4 . We see that the variances of the proposed method approached the CRLB for $N \in[4,10]$ while the PHD was inferior to the RPHD by at least $11 \mathrm{~dB}$ for $N \geq 4$.

\section{CONCLUSIONS}

Linear prediction based least squares approach has been investigated for estimating the frequency of a single sinusoid embedded in white noise. A new closed form frequency estimator for a noisy real-valued tone has been devised via minim izing a constrained least square cost function. The frequency estimator is computationally attractive and is simple to implement either in batch or recursive mode. The relationship of the devised algorithm with the Pisarenko's method is also explored. It is shown that the proposed method is generally superior to the Pisarenko harmonic decomposer and can give a very good estimation performance when the observation length is short and noise level is small.

\section{References}

[1] D.C.Rife and R.R.Boorstyn, "Single-tone parameter estimation from discrete-time observ ations," IEEE Trans. Inform. Theory, vol.20, no.5, pp.591-598, Sept. 1974

[2] S.M.Kay, Fundamentals of Statistical Signal Processing: Estimation Theory, Englewood Cliffs, N.: Prentice-Hall, 1993

[3] P.Stoica, "List of references on spectral line analysis," Signal Processing, vol.31, no.3, pp.329-340, April 1993

[4] B.G.Quinn and E.J.Hannan, The Estimation and Tracking of Frequency, New York: Cambridge University Press, 2001

[5] R.J.Kenefic and A.H.Nuttall, "Maximum likelihood estimation of the parameters of tone using real discrete data," IEEE Journal of Oceanic Engineering, vol.12, no.1, pp.279-280, 1987

[6] V.F.Pisarenko, "The retrieval of harmonics by linear prediction," Geophys. J. Roy. Astron. Soc., vol.33, pp.347-366, 1973

[7] H.Sakai, "Statistical analysis of Pisarenko's method for sinusoidal frequency estimation," IEEE Trans. Acoust., Speech, Signal Processing, vol.32, no.1, pp.95101, Feb. 1984

[8] M.Aktar, B.Sankur and Y.Istefanopulos, "Properties of the maximum likelihoodand Pisarenko spectral 
estimates," Signal Processing, vol.8, no.4, pp.401-413, July 1985

[9] P.Stoica and A.Nehorai, "Study of the statistical performance of the Pisarenko harmonic decomposition method," IEE Proceedings, vol.135, pt.F, no.2, pp.161168, April 1988

[10] A.Eriksson and P.Stoica, "On statistical analysis of Pisarenko tone frequency estimator," Signal Processing, vol.31, no.3, pp.349-353, April 1993

[11] E.Anarim and Y.Istefanopulos "Statistical analysis of Pisarenko type tone frequency estimator," Signal Processing, vol.24, no.3, pp.291-298, Sept. 1991

[12] P.A.Thompson, "An adaptive spectral analysis technique for unbiased frequency estimation in the presence of white noise," Proc. 13th Asilomar Conf. Circuits, Syst., Comput., Pacific Grove, CA, pp.529-533, Nov. 1979

[13] V.U.Reddy, B.Egardt and T.Kailath, "Least square type algorithm for adaptive implementation of Pisarenko's harmonic retrieval method," IEEE Trans. Acoust., Speech, Signal Processing, vol.30, no.6, pp.399-405, June 1982

[14] P.A.Regalia, "An adaptive unit-norm filter with applications to Karhunen-Loève transformation," IEEE Trans. Circuits and Systems, vol.37, no.5, pp.646-649, May 1990

[15] P.Stoica and A.Nehorai, "On linear prediction models constrained to have unit-modulus poles and their use for sinusoidal frequency estimation," IEEE Trans. Acoust., Speech, Signal Processing, vol.36, no.6, pp.940-942, June 1988

\section{Acknowledgement}

This work is supported by a research grant from City University of Hong Kong (Project No. 9030874).

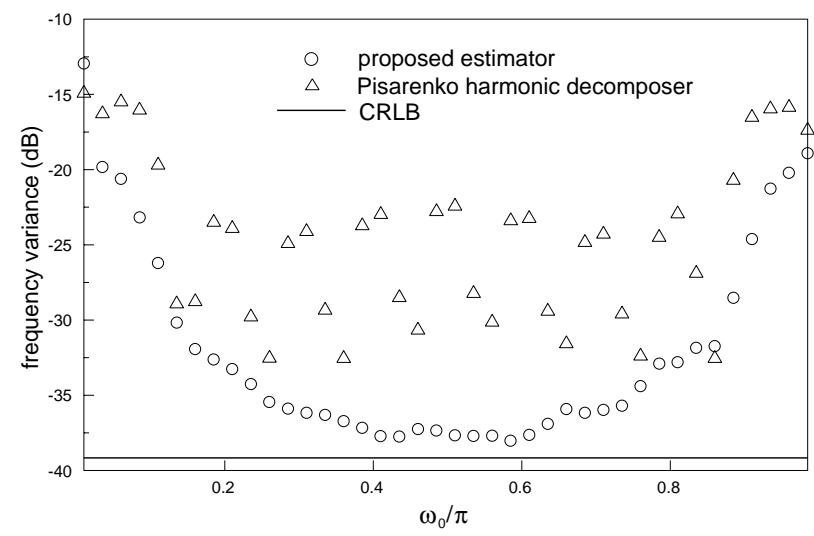

Figure 1: Frequency variances versus $\omega_{0}$ at $\mathrm{SNR}=20$ $\mathrm{dB} \& N=10$

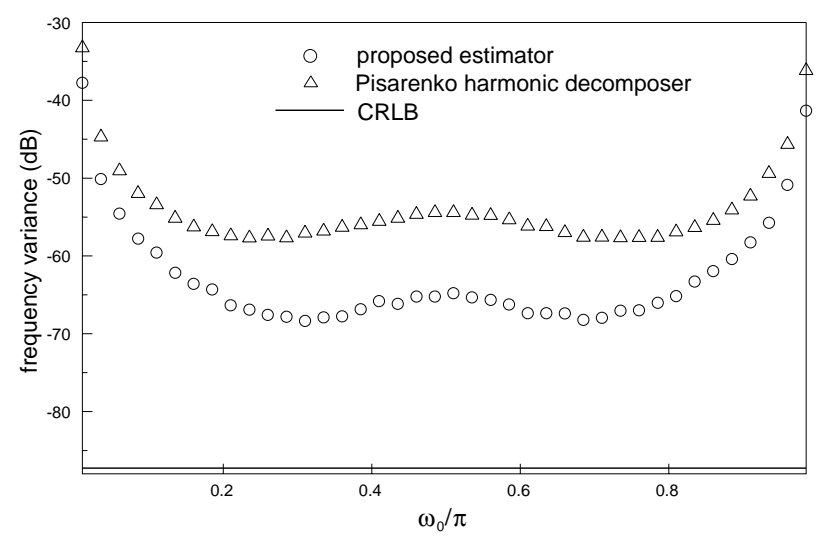

Figure 2: Frequency variances versus $\omega_{0}$ at $\mathrm{SNR}=20$ $\mathrm{dB} \& N=400$

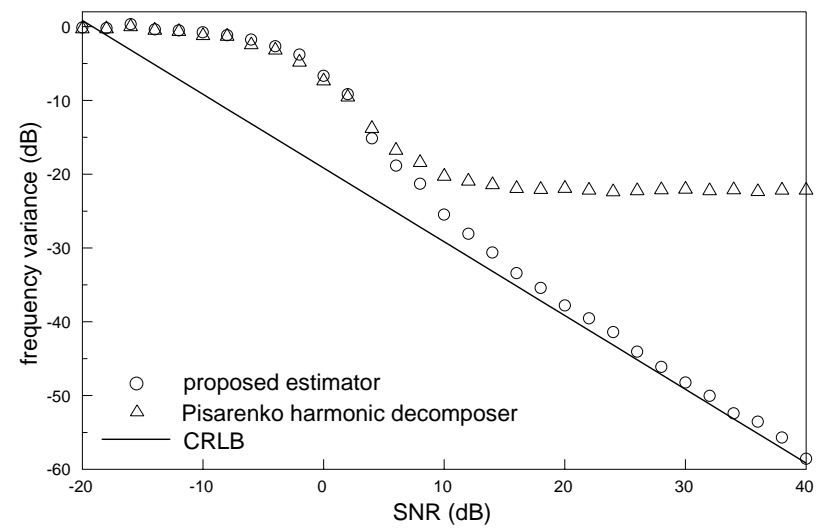

Figure 3: Frequency variances versus SNR at $\omega_{0}=0.5 \pi \& N=10$

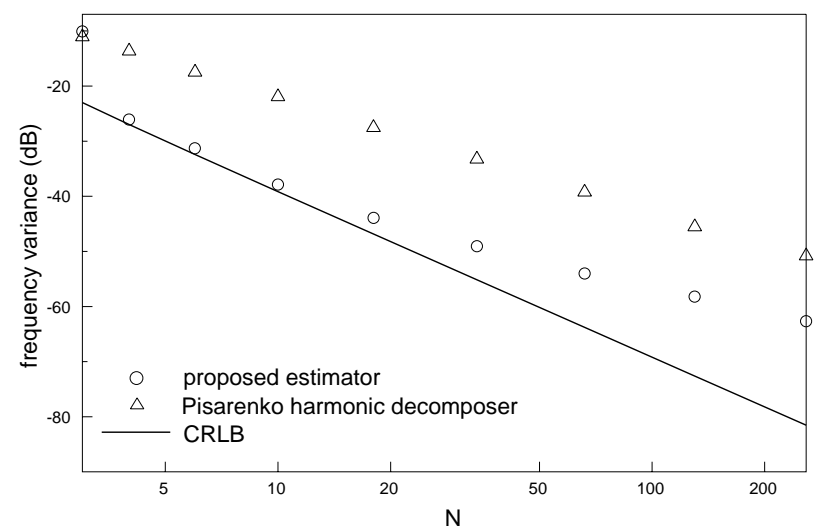

Figure 4: Frequency variances versus $N$ at $\omega_{0}=0.5 \pi$ $\& \mathrm{SNR}=20 \mathrm{~dB}$ 